\title{
Delay Analysis of STDMA in Grid Wireless Sensor Networks
}

\author{
Ichrak Amdouni*, Cedric Adjih*, Pascale Minet*, Thierry Plesse ${ }^{\dagger}$ \\ * Inria, France, Email: firstname.name@inria.fr \\ $\dagger$ DGA, Bruz, France, Email: thierry.plesse@intradef.gouv.fr
}

\begin{abstract}
TDMA (Time Division Multiple Access)) is known to be an affective method for energy saving in wireless sensor networks (WSNs). However, when any slot is exclusively reserved to one node, the TDMA-based protocols suffer from low-channel utilization and high data delivery delays. STDMA (Spatial reuse TDMA) avoids these drawbacks by allowing multiple nodes to access the same time slot provided that these nodes do not interfere. The objective of this paper is to evaluate the delay performance of STDMA assuming grid networks and a random ordering of the slots in the STDMA cycle. The evaluation is performed both with a stochastic analytical model and by experiments based on a known STDMA method called VCM. Results show that the random ordering performance is rather good. A framework for STDMA applied to general graphs under SINR interference model is also presented.
\end{abstract}

\section{INTRODUCTION}

\section{A. Context}

The objective of this paper is the delay analysis of STDMA based networks for military applications. Indeed, one of the main drivers for investigating wireless sensor networks is their promising military applications. Being equipped with appropriate sensors, these networks can enable enemy detection, biochemical area analysis, infantry protection, soldiers tracking, identification of enemy, force and analysis of their movement and progress [1].

Military applications have specific features implying many requirements for the wireless sensor network and the underlying operating protocols. Conventional platform-based military sensor systems are usually large and expensive. Thus, a dense deployment of sensors is required. Furthermore, sensors are usually battery operated and hence their energy is limited. When such sensors are deployed to monitor an enemy field, exchanging their battery is not possible. Thus, the solution is extending the lifetime of the network. Minimum delay guarantee and determinism is a critical issue for military WSNs. It is clearly the case when surveillance informations (alters, images or videos) must be transmitted to the brigade command post.

These requirements make the usage of the TDMA scheme at the MAC layer a good candidate.

In contrast to the contention-based protocols, TDMA-based solutions establish a schedule, called frame or cycle, where each node is assigned one (or possibly multiple) slots. By letting nodes turn-off their radios alternately, TDMA-based protocols significantly reduce collisions and achieve energy savings. Traditionally ([2], [3]), each node is assigned a fixed slot to transmit one message in each cycle. Nodes transmit on their assigned slots and wake up to receive in the slots of their neighbors.

\section{B. Delays in TDMA}

To prevent radio transmissions from interfering with each other, TDMA scheme prevents nodes (all or those that are in range) to transmit in the same slot. This usually requires a cycle with a large number of slots, leading to a significant message delay and a poor channel utilization. Consequently, this pure TDMA approach is unsuitable for delay sensitive applications and dense WSNs. STDMA (Spatial reuse TDMA) has been proposed to avoid the slots underutilization by allowing non interfering nodes to use the same time slot [4], [5], [13], [14]. Most existing works attempt to minimize the schedule length [4], [5].

However, this still not sufficient for minimal delays guarantee. Indeed, the order of medium access is also crucial. While routing protocols address the delay issues by choosing the shortest path, if nodes access their assigned slots in the opposite order of their appearance in the path, each time a node transmits a packet, the destination will be able to forward this packet only of the following cycle. This issue is know as "slot misordering problem" [12].

Two categories of solutions are proposed to address this problem: the first one assumes that routing is given and optimizes the scheduling accordingly. Inversely, the second one deduces the routing from a given scheduling. Examples of the first category include [6], [7], [8], [9], [10]. In [6], authors present an integer linear programming (ILP) formulation of the minimum latency link scheduling problem. Assuming that routes are given, the model is able to find the slot used by each link while minimizing the latency. We believe that the linear programming approach is not suitable for large networks. Solutions [8], [9], [10] assume that a data gathering tree is given. They ensure that any node has a slot earlier than its parent in the routing tree. Hence only one cycle is required for data aggregation. For instance, DMAC [9] allocates slots to nodes based on their positions in the data gathering tree. Nodes at the deepest levels in the tree are assigned the earliest slots.

In the second category, we can cite for instance $\mathrm{Yu}$ et al. [11], [25]. In [25], each link $(i, j)$ is assigned a cost that is equal to the time difference between slots assigned to nodes $i$ and $j$ respectively. The advantage of this solution is that 
all routes (from any node to another) are possible without scheduling change, but on the other hand nothing makes them energy-efficient.

In this paper, we consider the general case where a random order of the slots is applied independently from the joint routing protocol, and we evaluate the delays.

\section{Objective and Methodology}

The objective of this paper is the analysis of the delay performance assuming STDMA-based medium access scheme in a grid network. We assume that nodes are assigned slots and these slots are randomly ordered in the cycle. We first start by determining the obtained delays using a stochastic analytical model (Section III). Then, our aim is to compare this theoretical result with practical results. Thus, we need a practical slot assignment method. For our case, we base our comparison on a previous algorithm called VCM [15] (Section IV-B) which is based on graph coloring. Hence, we assume that the grid is colored with VCM where one color is assimilated to one slot. These slots are randomly ordered in the cycle. We consider in addition, that at the routing level, either greedy routing or shortest-delay routing is applied. We compute the obtained delays (Section IV-B) and compare them with the delays obtained with the stochastic model.

In the first part of this paper, we consider a grid network because such a regular deployment provides an optimal coverage [19], [20]. Furthermore, from the research point of view, working on grids can be a first step towards general graphs as it is easier especially for theoretical studies. This is what we will prove in Section $\mathrm{V}$; we will show that starting from a general graph, we can transform it on a grid and we provide a framework for applying STDMA for this grid while assuming a realistic SINR interference model.

\section{System Model And ASSUMPTIONS}

The system model considered in this paper is described in this section.

\section{A. A Framework for STDMA}

We consider a dense grid WSN. Each node is assigned a time slot for data transmissions. These slots are obtained by applying a given coloring algorithm. Generally, a slot assignment algorithm based on graph coloring works as follows. The graph is colored such that no two nodes that have the same color interfere. As an example, let us consider the linear network depicted in Figure 1(a). Assuming that interferences are limited to 2 hops, the same color is assigned to nodes $A$ and $D$, while a second color is assigned to nodes $B$ and $E$ and a third color is assigned to nodes $C$ and $F$ because these couples of nodes are 3 hops away. Consequently, they can transmit in the same slot without interfering (see cycle in Figure 1(b)).

The slots form the activity period in the STDMA cycle while the remaining part corresponds to the inactivity period where all nodes turn off their radio and save energy. Consequently, this scheme avoids energy wasted in idle listening and collisions. Finally, the global cycle is repeated in time.

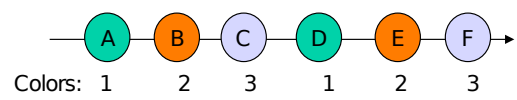

(a) Sample linear network.

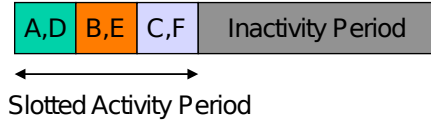

(b) STDMA cycle.

Fig. 1. Example of a network and the STDMA cycle associated.

\section{B. Assumptions}

- Application: We consider a military surveillance application where sensors monitor a region and transmit periodic measures or alarms to the sink (which can occupy any position in the grid). Long range communications are assumed; multi-hop routing is needed to deliver data to the sink. We suppose that data can be aggregated at any intermediate node and that packets are small. Hence, it becomes realistic to assume that any node is assigned a single time slot and that this time slot suffices for all its transmissions.

- Transmission and Interference Models: As in several wireless network studies, we adopt a unit disk communication model [26]. Any node can communicate directly with all nodes within a disk centered at itself with a communication range denoted $R$. In Section V, we will consider the SINR model.

- STDMA cycle inactivity: In performance evaluation sections, for simplicity, we assume a STDMA cycle without inactivity period, and the delay is expressed as the number of slots necessary for a packet to travel from a source to a destination $^{1}$ (as in Equation 2).

- One-Hop Delay: Let $D(i, j)$ be the induced one-hop delay for a packet of node $i$ that is further relayed by node $j$. Assume that node $i$ is assigned the slot $s_{i}$ while $j$ is assigned the slot $s_{j}$. Hence, $D(i, j)$ is equal to the difference between the slot where $i$ starts transmitting and the slot $s_{j}$ where the node $j$ relays the last bit of the packet of $i$. Let $S$ be the total number of slots. Like in [25] and assuming that there is no inactivity period, we have:

$$
D(i, j)=\left\{\begin{aligned}
s_{j}-s_{i} & \text { if } s_{j}>s_{i} \\
S+s_{j}-s_{i} & \text { if } s_{j}<s_{i}
\end{aligned}\right.
$$

- Route Delay: on a route (path) $\mathcal{P}$ of successive nodes $\left(v_{1}, v_{2}, v_{3}, \ldots, v_{k}\right)$, the delay is computed as the sum of successive one-hop delays except for the last node (which is the destination and does not relay the packet).

$$
D(\mathcal{P})=D\left(v_{1}, v_{2}\right)+D\left(v_{2}, v_{3}\right)+\ldots+D\left(v_{k-2}, v_{k-1}\right)
$$

- Normalized Delay per Range: it is the route delay with

\footnotetext{
${ }^{1}$ Notice that for a large delay $D$ of spanning several cycles of length $C$, the number of inactivity periods is directly estimated as $\lfloor D / C\rfloor$ with an error of at most 1 . Hence even with inactivity periods, for large $D / C$ mostly the value of the delay $D$ is of interest.
} 
distance normalized by radio range. In other words, if $i$ and $j$ are separated by geometric (Euclidean) distance $|i-j|$, and that path between them is $\mathcal{P}=(i, \ldots, j)$, hence with delay $D(\mathcal{P})$ slots, the normalized delay $D$ is:

$$
\bar{D}(\mathcal{P})=\frac{D(\mathcal{P})}{|i-j| / R}
$$

It represents the average delay, expressed in number of slots, necessary to progress by a distance equivalent to one radio range (on the route). This quantity takes into account both delay and geographic progress which makes it a good heuristic.

Remark 1: Notice that we have considered the coloring as the scheduling method, because the coloring is a good model for our problem statement and it allows us to have a practical algorithm for the numerical results. Otherwise, the theoretical results in Section III remain valid for any scheduling method assuming the same network model.

\section{Estimate of Normalized Delay PeR RANGe WITH RANDOM COLOR ORDERING}

We design a model to estimate the average normalized delay per range. Unlike previous works (such as [18], [21]), this model selects as relay the neighbor yielding the best normalized delay per range.

\section{A. Model}

We follow the approach commonly used in geographic routing studies, assuming that a route from a source to a destination is followed. Then the expected progress towards the destination is computed on one step at an intermediate relay node (as in [22] for instance). Greedy routing is assumed: but here, unlike geographic routing, the selected next hop is not the one that is the closest to the destination, but the one that minimizes the "normalized delay per range" (as in [23]).

Let $N$ be this intermediate node. Without loss of generality, we assume that its coordinates are $(0,0)$ and its color is 0 . We denote $n$ the radio range. Let $\mathcal{N}_{u}$ the set of neighbors of $N$ with x-coordinate $u$ where $u$ in an integer $\in[0, n]$. Let $y(u)$ be the maximum y-coordinate of these nodes. We have $y(u)=\left\lfloor\sqrt{n^{2}-u^{2}}\right\rfloor$. Also, asymptotically, we can write:

$$
y(u)=\sqrt{n^{2}-u^{2}}+O(1)
$$

The colors of these nodes are denoted $c_{u,-y(u)}, \ldots, c_{u, 1}, c_{u, 2}, \ldots, c_{u, y(u)}$. Figure 2 illustrates an example. For clarity reasons, we only present the colors of nodes in the quarter of the disk.

We assume the following approximations: 1) that the destination lies on the $\mathrm{x}$-axis (towards infinity), 2) that the colors are i.i.d (independent and identically distributed) random real values (not integer) uniformly distributed in the interval $[\alpha, \beta]$. We select as next hop the neighbor with coordinates $(u, i)$ that minimizes the "normalized delay per range", that is the quantity $\frac{c_{u \cdot i}}{\frac{u}{n}}$.

Let $Z \stackrel{\frac{u}{n}}{=} \max _{(u, i) \in N_{u}} \frac{c_{u . i} n}{u}$ be this quantity. It is a random variable, and we show that:

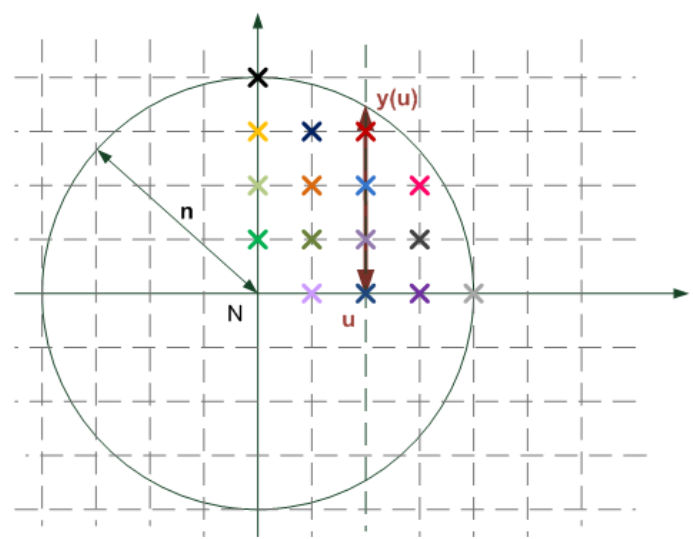

Fig. 2. Figure illustrating the model used.

Property 1: For $n \rightarrow \infty$, the distribution of $Z$ is approximated by a shifted exponential distribution that has expected value of $\frac{3 \alpha \pi}{4}+\frac{3(\beta-\alpha)}{2 n^{2}}$.

\section{B. Computation of the Normalized Delay per Range}

Let now prove Property 1 . We introduce intermediate random variables:

- $Y_{u, i} \triangleq \frac{c_{u, i} n}{u}$ is the normalized delay per range when selecting neighbor $(u, i)$ with color $c_{u, i}$. Because $c_{u, i}$ is uniformly distributed in $[\alpha, \beta]$, its cumulative distribution function is:

$$
F_{Y_{u, i}}(x)=\operatorname{Pr}\left(Y_{u, i} \leq x\right)=\sigma\left(\frac{\frac{x u}{n}-\alpha}{\beta-\alpha}\right)
$$

with $\sigma$ a "bounding function", defined as follows:

$$
\sigma(x)= \begin{cases}x & \text { for } 0 \leq x \leq 1 \\ 0 & \text { for } x<0 \\ 1, & \text { for } x>1\end{cases}
$$

- $X_{u} \triangleq \min _{i=-y(u), \ldots,+y(u)} Y_{u, i}$ is the normalized delay per range when choosing the best neighbor with $\mathrm{x}$-coordinate $u$. Its distribution verifies:

$$
F_{X_{u}}(x)=\operatorname{Pr}\left(X_{u} \leq x\right)=1-\left(1-\operatorname{Pr}\left(Y_{u, 0} \leq x\right)\right)^{(2 y(u)+1)}
$$

- The random variable $Z$ can also be expressed as a minimum of minima: $Z=\min _{u \in\{0,1, \ldots . n\}} X_{u}$. Using Equations (5) and (6), it is possible to express its cumulative distribution $F_{Z}(x)$ as:

$$
\begin{aligned}
F_{Z}(x) & =\operatorname{Pr}(Z \leq x) \\
& =1-\prod_{u=0}^{n}\left(1-\operatorname{Pr}\left(X_{u} \leq x\right)\right)
\end{aligned}
$$

Hence,

$$
\log \left(1-F_{Z}(x)\right)=\sum_{u=0}^{n}(2 y(u)+1) \log \left(1-\sigma\left(\frac{\frac{x u}{n}-\alpha}{\beta-\alpha}\right)\right)
$$


If we approximate $\log (1-a) \approx-a$ for $a$ near 0 (also an upper bound in all cases), and if we ignore bounding effects of $\sigma$, we can approximate $Z$ by $\tilde{Z}$, with a close distribution function (when $n \rightarrow \infty$ ):

$$
\log \left(1-F_{\tilde{Z}}(x)\right)=-\sum_{u=0}^{n}(2 y(u)+1) \frac{\frac{x u}{n}-\alpha}{\beta-\alpha}
$$

Using Equation 4, we have:

$$
\begin{aligned}
\log \left(1-F_{\tilde{Z}}(x)\right)= & -\frac{2}{\beta-\alpha} \times \\
& \left(\frac{x}{n} \sum_{u=1}^{n} u \sqrt{n^{2}-u^{2}}\right. \\
& \left.-\alpha \sum_{u=1}^{n} \sqrt{n^{2}-u^{2}}+O(n)\right)
\end{aligned}
$$

Now we introduce the following approximations:

$$
\begin{gathered}
\sum_{u=0}^{n} \sqrt{n^{2}-u^{2}}=\frac{\pi n^{2}}{4}+O(n) \\
\sum_{u=0}^{n} u \sqrt{n^{2}-u^{2}}=\frac{n^{3}}{3}+O\left(n^{2}\right)
\end{gathered}
$$

Indeed, these are sum of (bounded) functions, respectively: non-decreasing and with one unique maximum (increasing then decreasing). It is easy to bound them with integrals:

$$
a_{1} \leq \sum_{u=0}^{n} \sqrt{n^{2}-u^{2}} \leq a_{2}
$$

where:

$$
\left\{\begin{array}{l}
a_{1}=\left(\int_{0}^{n} \sqrt{n^{2}-u^{2}} \mathrm{~d} u\right)-n \\
a_{2}=\left(\int_{0}^{n} \sqrt{n^{2}-u^{2}} \mathrm{~d} u\right)+n \quad\left(\text { since } \sqrt{n^{2}-u^{2}} \leq n\right)
\end{array}\right.
$$

and:

$$
b_{1} \leq \sum_{u=0}^{n} u \sqrt{n^{2}-u^{2}} \leq b_{2}
$$

where:

$$
\left\{\begin{array}{l}
b_{1}=\left(\int_{0}^{n} u \sqrt{n^{2}-u^{2}} \mathrm{~d} u\right)-3 n^{2} \\
b_{2}=\left(\int_{0}^{n} u \sqrt{n^{2}-u^{2}} \mathrm{~d} u\right)+3 n^{2}
\end{array}\right.
$$

Hence,

$$
\log \left(1-F_{\tilde{Z}}(x)\right)=-\frac{2 n^{2}}{\beta-\alpha}\left(\frac{x}{3}-\alpha \frac{\pi}{4}+O\left(\frac{1}{n}\right)\right)
$$

Further ignoring the $O\left(\frac{1}{n}\right)$, we approximate $\tilde{Z}$ by $\widehat{Z}$ with:

$$
\operatorname{Pr}(\widehat{Z} \leq x)=1-\exp \left[-\frac{2 n^{2}}{3(\beta-\alpha)}\left(x-\frac{3 \alpha \pi}{4}\right)\right]
$$

Equation 9 is meaningful for $x \geq \frac{3 \alpha \pi}{4}$. Equation 9, corresponds to a variable $\widehat{Z}-\frac{3 \alpha \pi}{4}$ exponentially distributed with parameter $\lambda=\frac{2 n^{2}}{3(\beta-\alpha)}$. Therefore, the expectation of $\widehat{Z}, E(\widehat{Z})$ is:

$$
E(\widehat{Z})=\frac{3 \alpha \pi}{4}+\frac{1}{\lambda}=\frac{3 \alpha \pi}{4}+\frac{3(\beta-\alpha)}{2 n^{2}}
$$

$\widehat{Z}$ is an approximation of $Z$ and hence Property 1 .

\section{The Expected Normalized Delay per Range Assuming VCM Coloring}

Property 1 gives a general formula of the expected normalized delay per range, it does not assume a particular graph coloring algorithm. To obtain numerical results, we select a practical coloring algorithm by assuming that our network is colored with VCM (more details about this algorithm are in Section IV-B). With this scenario, the expected normalized delay per range is given by Property 2 :

Property 2: An estimate of the normalized delay per range is $\frac{3}{2} \theta+\frac{3}{4} \pi$, when the radio range $R$ grows and the number of colors is $\theta R^{2}$, where $\theta=\frac{\sqrt{3}}{2} h^{2}$, when VCM h-hop coloring is applied $^{2}$ ).

Proof: According to Property 2, the normalized delay per range is equal to: $\frac{3 \alpha \pi}{4}+\frac{3(\beta-\alpha)}{2 n^{2}}$. Applying VCM coloring yields, asymptotically, a cycle of length $\theta n^{2}$, where $n=R$ is the transmission range and $\theta=\frac{\sqrt{3}}{2} h^{2}$ (see Property 3). Hence, if we take $\alpha=1$ and $\beta=\theta n^{2}$, the normalized delay per range becomes: $\frac{3 \pi}{4}+\frac{3\left(\theta n^{2}-1\right)}{2 n^{2}}$. Asymptotically, we get: $\frac{3 \pi}{4}+\frac{3 \theta}{2}$

The implication of this result is rather strong: despite the fact that the number of colors grows as $R^{2}$, with random color ordering, to travel a distance of $K$ hops, the delay in "number of slots" stays equal to a constant $\left(\frac{3}{2} \theta+\frac{3}{4} \pi\right)$ multiplied by $K$. For example, for a 3 -hop coloring with $\operatorname{VCM}(h=3)$, the estimate of the normalized delay per range is $\approx 14.05 \ldots$ which is rather low.

\section{Experimental Validation of Asymptotic Expression for Nor- malized Delay per Range}

The goal of the present section is to compare the asymptotic expression of the normalized delay per range with actual results obtained by simulations of the exact modeled system.

\footnotetext{
${ }^{2}$ a h-hop coloring prevents nodes up to $h$ hops to use the same color.
} 


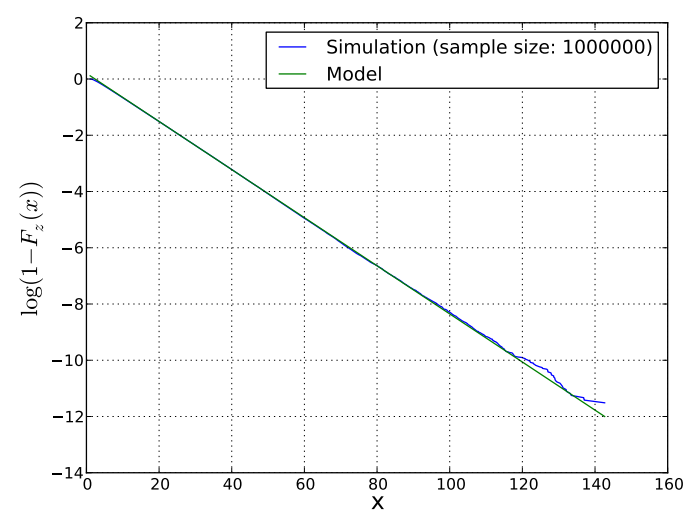

(a) Cumulative distribution in the form: $\log (1-F(x))$

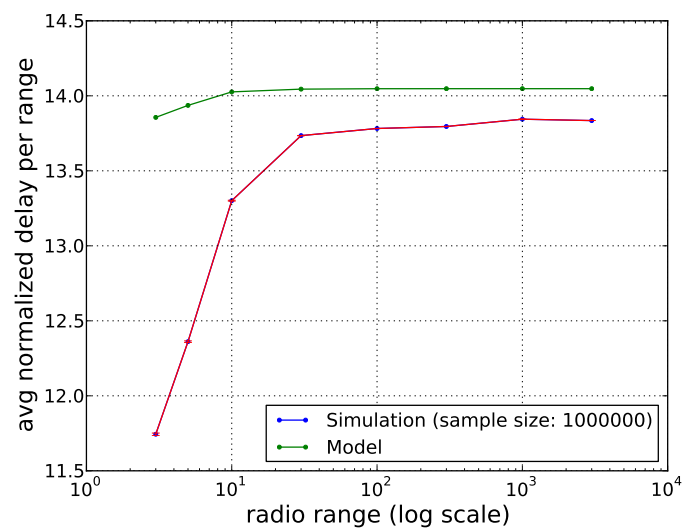

(b) Convergence to asymptotic expression (magnified: y-axis does not start from 0 )

Fig. 3. Experimental Validation of Model.

Figure 3(a) represents the results of computing the average value of normalized delay per range (as in Property 2) for a radio range of 1000 (from one million random draws).

As shown in the figure, parameters of the exponential distribution match very closely the actual cumulative distribution.

The complementary Figure 3(b) represents the convergence of the simulation results to the asymptotic expression. The chosen y-axis scale enhances the differences, but for a radio range equal to 3000 we witness a difference of only $1.5 \%$ between model and simulations.

These experimental results validate the convergence to the asymptotic value, and also some of the approximations that were made.

\section{ESTIMATE OF THE DELAYS WITH EXPERIMENTS BASED ON IRCO AND VCM}

This section assumes an arbitrary assignment of colors in the cycle. We assume that colors are obtained by VCM coloring. The objective is to evaluate the performance of the delay under such assumptions and compare these results with the model theoretical results.

\section{A. VCM: Vector-based Coloring Method}

VCM [15], [16] is a graph coloring algorithm dedicated to dense grid networks. The intuitive idea of VCM is as follows. As the grid topology presents a regularity in terms of node positions, VCM produces a similar regularity in terms of colors and generates a color pattern that can be periodically reproduced to color the whole grid. An example of the coloring provided by VCM is given in Figure 4.

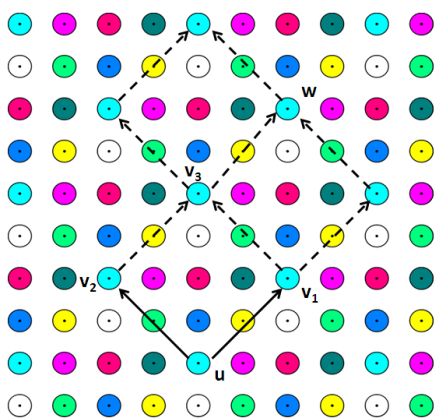

Fig. 4. An example of 3-hop coloring provided by VCM (no 1 or 2 or 3-hop neighbors share the same color.)

The color pattern is defined by two vectors $\left(u \vec{v}_{1}\right.$ and $u \vec{v}_{2}$ in Figure 4) called VCM generator vectors. VCM finds a color pattern that minimizes the number of colors used and hence minimizes the length of the TDMA cycle. The number of colors produced by VCM does not depend on the size of the grid, but on the transmission range of sensor nodes.

The schedule that VCM provides is optimal in terms of length when the transmission range tends to infinity. Indeed, we have proved in [16], that the number of colors is given by Property 3.

Property 3: (From [16]) The number of colors $n_{c}(R)$ of an optimal periodic $h$-hop coloring, including VCM, for a fixed $h$ verifies:

$n_{c}(R)=\theta R^{2}\left(1+O\left(\frac{1}{R}\right)\right)$, when $R \rightarrow \infty$, with $\theta=\frac{\sqrt{3}}{2} h^{2}$.

\section{B. Random Color Ordering and IRCO}

In addition to the assumptions presented in Section II, we consider a grid colored with VCM and we assume that the colors are randomly associated with slots. This assumption allows us to define a basic method for building the global cycle. We denote this method IRCO ("Iterated Random Color Ordering").

IRCO consists in randomly ordering colors of a single VCM cycle and then repeating successively this base VCM cycle several times in a row before the inactivity period. For instance, one IRCO cycle is a repetition of several identical VCM cycles necessary to allow all data from any source to reach its destination. Notice that IRCO deals with colors ordering and is independent from the routing adopted. In the following, we will evaluate IRCO with two routing strategies: shortestpath routing and greedy routing. 


\section{Routing}

Two routing strategies are used:

1) Shortest Delay Routing: Because the delay as defined in Section II is an additive metric, it is possible to compute the "shortest path" using classical algorithms (Bellman-Ford, Dijkstra) with this metric, as proposed for instance by [24]. The paths that are found are the shortest paths in terms of delay (expressed as in Equation 2) necessary to reach the destination. Note that equivalently, they yield the lowest normalized delay per range computed over the whole route.

2) Greedy Routing Heuristic: One possible strategy for routing on a colored grid, is the following: at every hop, decide what would be the next hop, based only on local information (and not global information). This is a strategy derived from geographic routing. For delay-aware routing, it is better to select a relay node presenting the best compromise between delay and progress. Such a greedy heuristic is proposed, for instance, in CMAC [23]: the selected next-hop is the one that yields the lowest ratio "delay"/"progress towards the destination". We can adopt this heuristic in this context of grid networks and predefined STDMA. Note that this is equivalent to yielding the lowest normalized delay per range computed on one hop.

\section{Experimental Results}

The parameters of simulation are summarized in Table I.

\begin{tabular}{|l|l|}
\hline Nb simulations & 100 random orderings with same VCM vectors \\
\hline Radio range & from 1 to 7 with step $1 / 4$ \\
\hline Routing strategy & shortest-delay path or greedy heuristic \\
\hline Size of the grid & $L \times L$ with $L=601$ \\
\hline \hline Sources for Greedy routing & $\begin{array}{l}100 \text { random nodes at distance } D \text { with } 0.9 L \leq \\
D \leq L\end{array}$ \\
\hline $\begin{array}{l}\text { Sources for Shortest-Delay } \\
\text { Path routing }\end{array}$ & all nodes at distance $D$ with $0.9 L \leq D \leq L$ \\
\hline
\end{tabular}

The two simulated strategies are the greedy routing and the shortest-delay path denoted respectively "Sim. greedy endto-end" and "Sim. shortest-delay path end-to-end". For each strategy, source nodes were selected according to Table I. The path from each source to the center point $(0,0)$ was computed. We first compute the total end-to-end delay (expressed in slots). Then, we obtain the normalized delay per range by dividing this delay by the geometric distance (normalized by radio range). An average was taken on all selected nodes, and on multiple simulations. We also compute the asymptotic estimate of the model given by Property 2 .

We first evaluate the normalized delay for one step. Figure 5 illustrates the average normalized delay per range computed by exactly simulating the scenario of the model, and also the simulations of the greedy routing ("Sim.greedy end-to-end strategy"). The observed normalized delay is similar for both methods. This result validates the method and scenario adopted by our model to compute the average normalized delay per range.

Figure 6 compares the end to end delays obtained by the model, the "Sim. shortest-delay path end-to-end" and "Sim. greedy end-to-end".

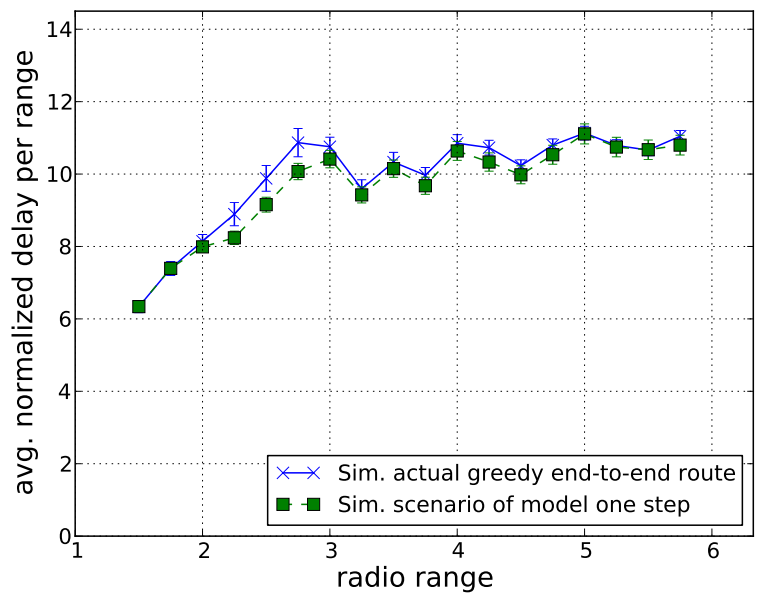

Fig. 5. Normalized delay in greedy routing and model.

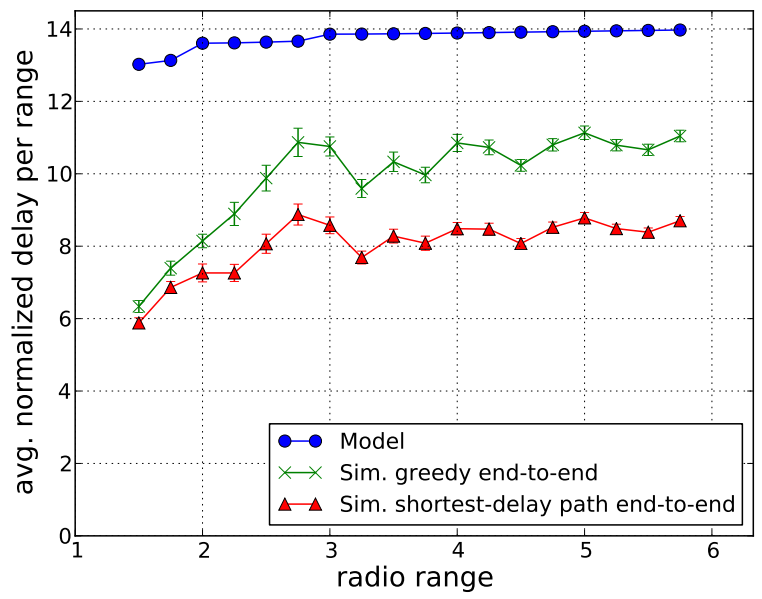

Fig. 6. Normalized delay for random color orderings.

The main result is that the normalized delay per range increases only slowly with the radio range (although the number of colors increases as the square of the range) and is quickly plateauing (as predicted by the model in Section III). This illustrates that the random ordering of colors yields a bounded normalized delay even when the number of colors increases to infinity. In other words, random ordering of colors is an optimal ordering when the radio range increases.

Furthermore, for both cases of greedy routing and shortestdelay path, the normalized delay per range is below 12 (and the asymptotic estimate is 14.0..). Knowing that a loose lower bound is 1 , this shows that the obtained delay is within an order of magnitude of an optimal STDMA.

Comparing shortest-delay path and greedy routing, we see that shortest-delay path gives approximately $25 \%$ better performance than greedy routing. The conclusion is that greedy routing performs quite well, if we take into account the fact that it does require only local information. Indeed, shortest- 
delay path, requires, of course, a shortest-path computation of every destination, which is cumbersome for WSNs, in a scenario where the destination(s) are not known in advance. Notice here the irregularity of the curves. This is explained by the fact that colors by radio range area vary following the same irregularity. Indeed, when we plot the number of colors divided by the area of the disk of radio range $\left(\pi R^{2}\right)$, we also see the same irregularity.

\section{E. Discussion about IRCO Performance}

The expected performance of IRCO is rather good, as deduced from results. However, random ordering suffers from drawbacks. The dimensioning of the entire cycle necessary to allow all data from any source to reach its destination is critical. It depends on the set of destinations and could be estimated from experimental results seen above. The drawback of IRCO is that either dimensioning is only estimated, potentially losing determinism (guarantees of arrival within one IRCO cycle), or the dimensioning requires pre-computing in advance all routes. In addition, all nodes are potential relays, and therefore should be awake on their neighbors slots. Hence, it is worth addressing the problem of slot ordering regarding the application and routes for instance as done in [12].

\section{From General to Grid Networks}

So far, we have assumed a grid network. In this section, we prove that we can map a grid on a general graph and color the nodes of this general graph with a modified version of VCM based on the SINR.

\section{A. Mapping a Grid on a General Graph}

We assume that we have a general graph of a given density. We assume also that this graph is fully connected. We define a grid of cells such that:

- This grid contains all nodes.

- The grid step (the cell width) is $\leq$ the transmission range of nodes. It is normalized to 1 .

- We assume that the network is sufficiently dense to assume that each cell contains at least one node.

Given this cell grid, we proceed as follows:

- Instead of coloring nodes individually, our idea consists in coloring the cells using $V C M++$ : a modified version of VCM that is based on the SINR interference model (see section V-B). Hence, each cell has a color computed by VCM++. Cells having the same color are cells at the lattice formed by the generator vectors of $\mathrm{VCM}++$.

- Nodes inside one cell are assigned different colors.

- Hence, each cell has a slot called super-slot associated with its color. This slot itself is divided into sub-slots reserved to the colors of nodes inside the corresponding cell.

Figure 7 illustrates an example.

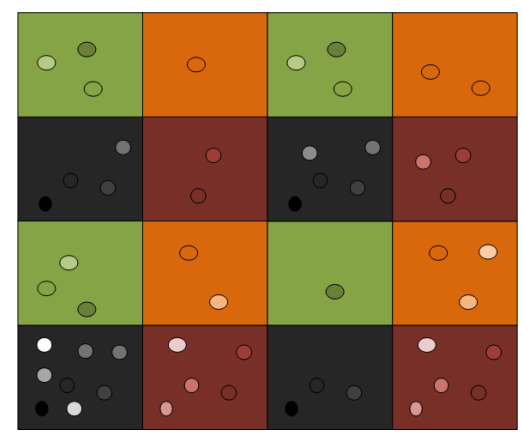

(a) Cell Grid

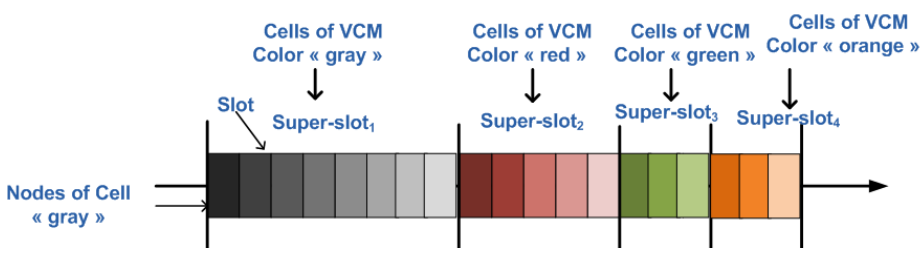

(b) Routes Cycle

Fig. 7. An illustrative example of a colored cell grid and the cycle obtained.

In this figure, we have 4 colors, hence 4 super-slots. The most populated cell with color orange contains 3 nodes, hence the super-slot orange is divided into 3 sub-slots.

Consequently, all nodes have colors and hence slots in the global STDMA cycle.

\section{B. VCM++: Adapting VCM to Color Cells using SINR Inter- ference Model}

The physical interference model is defined as follows [27].

Definition 1: Let $P$ the common transmission power at all nodes. Let $\left(X_{k}\right), k$ integer, be the set of nodes transmitting simultaneously. Let the constant $N$ be the background noise. A transmission from a node $X_{i}$ is successfully received by a node $X_{j}$ if:

$$
\frac{\frac{P}{\left|X_{i}-X_{j}\right|^{\alpha}}}{N+\sum_{k \neq i} \frac{P}{\left|X_{k}-X_{j}\right|^{\alpha}}} \geq \beta
$$

where $\left|X_{i}-X_{j}\right|$ denotes the distance between any two nodes $X_{i}$ and $X_{j}$ and $\beta$ a constant threshold.

Many works dealt with the scheduling in lattice based on SINR interference model (e.g. [14], [28]). In our work, concurrent transmissions are at the lattice generated by $\mathrm{VCM}++$ vectors. The aim of this section is to modify the method of vector search of VCM in case SINR interference model is considered. Let $\mathcal{L}\left(u_{1}, u_{2}\right)$ be the lattice generated by VCM++ generator vectors $u_{1}$ and $u_{2}: \mathcal{L}\left(u_{1}, u_{2}\right)=\left\{\left(a u_{1}+b u_{2}\right) \mid(a, b) \in \mathbb{Z}^{2}\right\}$. For any vector $u$, we define the cell $c(u)$ as: $c(u)=\{u+$ $\left.(x, y) \mid(x, y) \in[0,1]^{2}\right\} . u$ is called the coordinate of the cell. When we apply VCM++ to color the cells, all nodes in all cells of coordinates $w$ such that $w \in \mathcal{L}\left(u_{1}, u_{2}\right)$ will transmit at the same super-slot. Considering a transmission from node $(0,0)$ to node with coordinates $z$, the interference at node of 
coordinate $z$ in the worst case is:

$$
I(z)=\sum_{w \in \mathcal{L}\left(u_{1}, u_{2}\right) \backslash\{(0,0)\}} \max _{t \in c(w)} P|t-z|^{-\alpha}
$$

The associated SINR $S_{\min }(w)$ computed at any cell $c(v)$ upon a reception from the cell $c(0)$ is:

$$
S_{\min }(v)=\min _{\substack{t_{u} \in c(0) \\ t_{v} \in c(v)}} \frac{P\left|t_{u}-t_{v}\right|^{-\alpha}}{I(v)+N}
$$

The transmission from $c(0)$ to $c(v)$ is successful if and only if: $S_{\min }(v) \geq \beta$. Then, to determine the successful transmissions from $c(0)$, we search the largest disk of radius $D_{u_{1}, u_{2}}$ where every cell can receive successfully from $c(0)$. Hence, $D_{u_{1}, u_{2}}=\max \left\{|v|: v \in \mathbb{Z}^{2}, \forall w \in \mathbb{Z}^{2}:|w|>\right.$ $\left.|v| \Longrightarrow S_{\min }(w)<\beta\right\}$.

Finally, we can select $u_{1}$ and $u_{2}$ as:

$$
u_{1}, u_{2}=\underset{\left(u_{1}, u_{2}\right)}{\operatorname{argmax}} \frac{\pi D_{u_{1}, u_{2}}^{2}}{\operatorname{det}\left(\mathrm{u}_{1}, \mathrm{u}_{2}\right)}
$$

With this formula, we ensure a tradeoff between the number of successful transmissions and the total number of colors.

\section{CONCLUSION}

This paper addressed the delay evaluation in STDMA systems. We first designed a stochastic model to evaluate the normalized delay per range when no specific order of the slots is required. Then, we conducted experiments with a known coloring algorithm and both shortest path and greedy routing. Comparison results validated the model. Also, we noticed that the delay obtained with the random ordering are rather good. However, we argue that such ordering is not aware about routing neither application to allow an efficient dimensioning of the global STDMA cycle. We also provided a method to map a grid to a general graph using a new version of VCM based on the SINR.

\section{ACKNOWLEDGEMENT}

This work has been partly supported by DGA/ASTRID/ANR-11-ASTR-0033 under the GETRF project [17].

\section{REFERENCES}

[1] Winkler M., Tuchs K., Hughes K., and Barclay G., Theoretical and practical aspects of military wireless sensor networks, Journal of Telecommunications and Information Technology.

[2] Van Hoesel L, Havinga P. A Lightweight medium access protocol (LMAC) for wireless sensor networks: Reducing Preamble Transmissions and Transceiver State Switch, Proceedings of the 1st International Workshop on Networked Sensing Systems (INSS), Tokyo, Yapan, 2004: 205-208.

[3] Rajendran V, Obraczka K, Garcia-Luna-Aceves JJ., Energy-efficient collision-free medium access control for wireless sensor networks, Proceedings of the first international conference on Embedded networked sensor systems SenSys 03, Los Angeles, CA, November 2003; 12 (1): 181-192, DOI: 10.1145/958511.958513.

[4] Incel D.O., Ghosh A., Krishnamachari B., Chintalapudi K., Fast data collection in treebased wireless sensor networks, IEEE Transactions on Mobile Computing, 2009.
[5] Ergen S.C., Varaiya P., TDMA scheduling algorithms for wireless sensor networks, Wireless Networks, May 2010.

[6] Cheng M.X., Gong X., Xu Y., Cai L., Link activity scheduling for minimum end-to-end latency in multihop wireless sensor networks, in IEEE Globecom'11, 2011.

[7] Cheng M.X.,Ye Q., Cai L., Cross-Layer Schemes for reducing Delay in Multi-hop Wireless Networks, IEEE Transactions on Wireless Communications, vol. 12, no. 2, pp. 928-937, February 2013.

[8] Chatterjee P., Da N., A cross-layer distributed TDMA scheduling for data gathering with minimum latency in wireless sensor networks, Wireless Communication, Vehicular Technology, Information Theory and Aerospace \& Electronic Systems Technology, May 2009.

[9] Lu G., Krishnamachari B., Raghavendra C., An Adaptive Energy-Efficient and Low-Latency MAC for Data Gathering in Wireless Sensor Networks, Parallel and Distributed Processing Symposium, April 2004.

[10] Amdouni I., Minet P., Adjih C., Adaptivity of a Coloring Algorithm to Unreliable Communications for Data Gathering in Wireless Sensor Networks, International Journal of Digital Information and Wireless Communications (IJDIWC), vol. 3, pp. 61-74, Avril 2013.

[11] Deng X., Yang Y., Cluster Communication Synchronization in DelaySensitive Wireless Sensor Networks, DCOSS 2013, Cambridge 2013.

[12] Amdouni I., Adjih C., Minet P.: Delay and Energy-Efficient STDMA for Grid Wireless Sensor Networks: ORCHID, IEEE 11th International Conference on Mobile Ad Hoc and Sensor Systems (MASS) 2014.

[13] Nelson R.; Kleinrock, L., Spatial TDMA: A Collision-Free Multihop Channel Access Protocol, IEEE Transactions on Communications, vol. 33, no. 9, pp. 934-944, September 1985.

[14] Prabh K. S., Near-Optimal Distributed Scheduling Algorithms for Regular Wireless Sensor Networks, CoRR Journal, 2012.

[15] Adjih C., Amdouni I., Minet P., VCM: the vector-based coloring method for grid wireless ad hoc and sensor networks, MSWIM 2012, October 2012.

[16] Amdouni I., Adjih C., Minet P., On the Coloring of Grid Wireless Sensor Networks: the Vector-Based Coloring Method, Inria Research Report, October 2011

[17] http://getrf.gforge.inria.fr/

[18] Zorzi M., Rao Ramesh R., Geographic Random Forwarding (GeRaF) for Ad Hoc and Sensor Networks: Multihop Performance, IEEE Transactions on Mobile Computing, October 2003.

[19] Baumgartner T.; Fekete S.P.; Kamphans T.; Kröller A., Pagel M., Hallway Monitoring: Distributed Data Processing with Wireless Sensor Networks, REALWSN, December 2010.

[20] Chakrabarty K.; Iyengar S. S.; Qi H. R. and Cho E., Grid Coverage for Surveillance and Target Location in Distributed Sensor Networks, IEEE Transactions on Computers, vol. 51, pp. 1448-1453, 2002.

[21] Ma D., Er M.J., Wang B., Lim H.B., K-hop statistics in wireless sensor networks, ISSNIP, December 2009.

[22] Kleinrock L., Silvester J., Spatial Reuse in Multihop Packet Radio Networks, IEEE, January 1987.

[23] Liu S., Fan K.W., Sinha P., CMAC: An Energy Efficient MAC Layer Protocol Using Convergent Packet Forwarding for Wireless Sensor Networks, SECON'07, 2007.

[24] Joohwan K., Xiaojun L., Ness B. S., Optimal Anycast Technique for Delay-sensitive Energy-constrained Asynchronous Sensor Networks, IEEE/ACM Trans. Netw., April 2011.

[25] Yu F., Wu T., Biswas S., Routing with Minimized Slot Misordering for Delay Mitigation in TDMA based Sensor Networks, Third International Conference on Networking and Services (ICNS 2007), June 2007.

[26] Gräf A., Stumpf M., Weissenfels G., On coloring unit disk graphs, Algorithmica, 20(3), pp. 277-293, 1998.

[27] Gupta P.; Kumar P.R., The capacity of wireless networks, IEEE Transactions on Information Theory, vol. 46, pp. 388-404, March 2000.

[28] Haenggi M., Interference in Lattice Networks, http://arxiv.org/abs/1004.0027 